\title{
La perfection et la défaillance. Le problème de l'accès du sujet au salut dans le christianisme antique (III $-\mathrm{V}^{\mathrm{e}}$ siècle)
}

Thèse de doctorat de philosophie, sous la direction de Michel Senellart, ENS de Lyon, soutenue le 23 juin 2014.

Anthony Manicki

\author{
(2) OpenEdition \\ Journals \\ Édition électronique \\ URL : https://journals.openedition.org/cem/13531 \\ DOI : 10.4000/cem. 13531 \\ ISSN : 1954-3093 \\ Éditeur \\ Centre d'études médiévales Saint-Germain d'Auxerre
}

Référence électronique

Anthony Manicki, «La perfection et la défaillance. Le problème de l'accès du sujet au salut dans le christianisme antique (III-veve siècle) », Bulletin du centre d'études médiévales d'Auxerre / BUCEMA [En ligne], 18.2 | 2014, mis en ligne le 19 décembre 2014, consulté le 22 septembre 2022. URL : http:// journals.openedition.org/cem/13531; DOI : https://doi.org/10.4000/cem.13531

Ce document a été généré automatiquement le 22 septembre 2022.

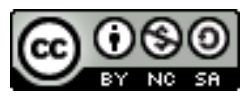

Creative Commons - Attribution - Pas d'Utilisation Commerciale - Partage dans les Mêmes Conditions 4.0 International - CC BY-NC-SA 4.0

https://creativecommons.org/licenses/by-nc-sa/4.0/ 


\section{La perfection et la défaillance. Le problème de l'accès du sujet au salut dans le christianisme antique $\left(\mathrm{III}^{\mathrm{e}}-\mathrm{V}^{\mathrm{e}}\right.$ siècle)}

Thèse de doctorat de philosophie, sous la direction de Michel Senellart, ENS de Lyon, soutenue le 23 juin 2014.

\section{Anthony Manicki}

Ce travail de doctorat se donnait au départ pour objectif de reconstruire les présupposés anthropologiques ${ }^{1}$ d'une forme de pouvoir typique du christianisme de l'Antiquité tardive et du Moyen Âge, le pastorat ${ }^{2}$. Partant du fait que toute constitution politique se fonde sur une anthropologie qui la légitime ${ }^{3}$, quelle conception de la nature humaine justifie un pouvoir qui se caractérise comme « un art de conduire, de diriger, de mener, de guider, de tenir en main, de manipuler les hommes, un art de les suivre pas à pas, un art qui a cette fonction de prendre en charge les hommes collectivement et individuellement tout au long de leur vie et à chaque pas de leur existence ${ }^{4}$ ? "

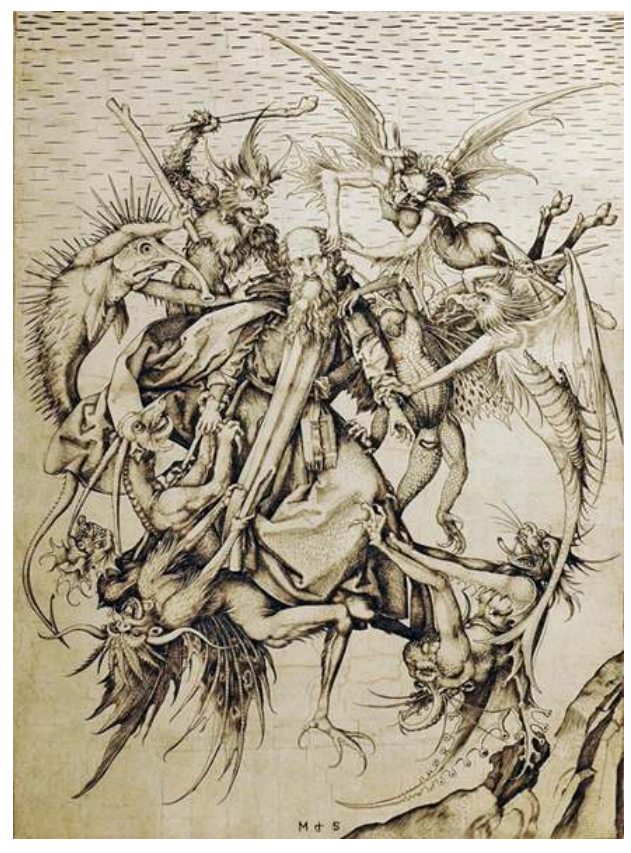




\section{"Perfectionnisme » et " défaillantisme », deux façons de penser la nature humaine dans le christianisme antique}

2 La littérature ascétique chrétienne des $\mathrm{III}^{\mathrm{e}}$ et $\mathrm{IV}^{\mathrm{e}}$ siècles se fonde sur une anthropologie héritée de la tradition philosophique antique ${ }^{5}$, qu'à la suite de Robert Markus nous avons nommée "perfectionnisme ${ }^{6}$. Elle accorde au sujet la capacité d'accéder à l'autonomie, à la perfection, au salut grâce à ses seules forces. Dans ce cadre, la difficulté de l'itinéraire spirituel du sujet provient soit du chemin emprunté, soit de sa mauvaise éducation, soit de sa mauvaise volonté. Mais rien, dans sa nature, ne l'empêche d'accéder à l'autonomie. Par conséquent, les formes de pouvoir qui régulent les rapports entre les hommes sont justifiées par leur manque d'éducation et non par leur nature. Dans l'idéal, si tous les hommes étaient bien éduqués, c'est-à-dire si tous suivaient les ordres de la raison, l'organisation politique serait superflue. L'existence d'un pouvoir coercitif est donc, dans ce cadre, une donnée factuelle ${ }^{7}$. Dans la Vie d'Antoine (vers 358), les scènes de lutte de l'ascète sont décrites par Athanase d'Alexandrie (vers 298-373) selon une structure fixe : Antoine est face à des démons qui tentent d'instiller en lui de mauvaises pensées ${ }^{8}$. Plusieurs fois, Athanase laisse entendre qu'un exercice patient sur soi peut conduire le sujet au salut ${ }^{9}$. Toute une série de textes ascétiques chrétiens antiques présentent la même idée: puisque l'ennemi affronté par le sujet dans le combat spirituel lui est extérieur, alors il est possible de remporter ce combat en usant des seules forces humaines ${ }^{10}$.

3 Une autre façon de penser le sujet humain se développe dans le christianisme au $\mathrm{V}^{\mathrm{e}}$ siècle. On peut lire les traces de ce processus chez Augustin (356-430). Évoquant son propre combat spirituel dans les Confessions (397-401), il affirme dans le livre VIII que son ardent désir d'obéir à Dieu s'est confronté à des désirs mauvais caractéristiques de son ancienne vie. La notion de «libido» permet de penser ces désirs. Plus tard, thématisant, dans la Cité de Dieu (413-415), les conséquences du péché originel, il utilise à nouveau cette notion pour désigner la force de résistance qui, en chaque homme, se confronte à sa raison. Là encore, la libido se réfère aux désirs mauvais qui s'opposent à la volonté divine et qui éloignent le sujet de Dieu. Mais derrière cette apparente continuité se cache une différence capitale. Dans le livre VIII des Confessions, la libido est pensée comme un produit contingent de la volonté individuelle ${ }^{11}$. Si Augustin est incapable d'obéir aux prescriptions divines, c'est dans la mesure où se sont sédimentées en lui des dispositions dont l'origine tient d'abord à une mauvaise orientation de la volonté et ensuite à l'habitude. Il lui suffirait alors de prendre l'habitude de bien vouloir pour produire en lui des dispositions vertueuses. Autrement dit, il est en son pouvoir d'être bon. Dans le livre XIV de la Cité de Dieu, la libido n'est plus pensée comme un produit de la volonté, mais comme une donnée essentielle de la nature humaine ${ }^{12}$. La force de résistance qui interdit à l'homme d'être maître de lui-même est pensée à partir du désir sexuel, l'autonomie de la libido impliquant alors l'hétéronomie caractéristique de la condition humaine ${ }^{13}$. Cette conception de la nature humaine, que nous avons nommée "défaillantisme", interdit au sujet d'accéder au salut par ses seules forces. Pour y parvenir, il a nécessairement besoin de la grâce divine. Clairement thématisé par Augustin durant la controverse pélagienne ${ }^{14}$, le défaillantisme justifie ontologiquement la soumission de l'homme: incapable de se gouverner lui-même, il doit se soumettre à des institutions censées compenser son infirmité. On peut ainsi voir 
dans l'anthropologie défaillantiste une justification du pouvoir pastoral. Cela étant dit, il nous a semblé que cette justification anthropologique du pouvoir pastoral impliquait une difficulté théorique extrêmement forte puisque les institutions devant compenser la faiblesse humaine étaient, en dernière instance, gérées par des hommes eux-mêmes structurellement défaillants. On trouve chez Jean Cassien (vers 360-435) plusieurs principes permettant, sinon de résoudre ce problème, du moins d'en minimiser la portée. Sont ainsi exposés, dans les Institutions cénobitiques, divers moyens permettant à l'individu de soigner sa faiblesse sans jamais cependant que ces soins le conduisent à une pleine et entière guérison ${ }^{15}$. Outre le dispositif associant l'examen de soi et l'aveu analysé par Foucault, l'un de ces moyens a particulièrement retenu notre attention à savoir l'exigence, au lieu de s'en tenir à sa propre faculté de juger, de suivre « la règle commune » [communis regula] ${ }^{16}$. On pourrait objecter à cela que la règle elle-même est un produit humain et qu'elle est donc potentiellement défaillante. Les auteurs chrétiens devaient donc trouver des moyens de présenter la règle comme une institution échappant, autant que faire se peut, à la faiblesse des hommes qui en furent les auteurs. Pour ce faire, ils purent tout d'abord souligner que la règle énonçait en réalité des préceptes contenus dans les Écritures auxquelles on attribuait une provenance divine ${ }^{17}$. Ils purent ensuite mettre en avant la sainteté de celui qui rédigea la règle et dont l'existence et la doctrine s'approchaient de la perfection ${ }^{18}$. Ils purent enfin affirmer que l'ancienneté de la règle était le signe de son efficacité ${ }^{19}$. Autrement dit, la littérature chrétienne présenta, dans le cadre du défaillantisme, bon nombre de réflexions traitant le problème, d'apparence insoluble, du gouvernement des hommes défaillants par d'autres hommes défaillants.

\section{Les conséquences de ces deux façons de penser le sujet humain}

Pour préciser les contours du perfectionnisme et du défaillantisme chrétiens, nous avons ensuite analysé les discours prenant pour objets deux formes de vie originales mises en place dans le christianisme à partir du III e siècle, l'érémitisme et le cénobitisme. Jusqu'à la fin du IV siècle, c'est à l'érémitisme qu'est accordée la plus grande dignité ${ }^{20}$. Ce jugement se fonde sur l'anthropologie perfectionniste. La capacité, pour le sujet, d'accéder au salut en comptant sur ses seules forces conduit à concevoir toute liaison au monde comme une compromission : si le sujet veut être parfait, il doit se défaire des relations sociales qui sont autant de liens l'empêchant de s'adonner à la seule activité légitime, le service de Dieu ${ }^{21}$. C'est tout le sens de la parole qui initie le parcours spirituel d'Antoine : «Si tu veux être parfait, va, vends tout ce que tu possèdes et donne-le aux pauvres, et viens, suis-moi, et tu auras un trésor dans les cieux ${ }^{22}$.»

$5 \quad \mathrm{Au}$ début $\mathrm{du} \mathrm{v}^{\mathrm{e}}$ siècle, la supériorité de l'érémitisme sur le cénobitisme est contestée. Si l'existence du solitaire est toujours admirée, certains doutent cependant que ce mode de vie soit fait pour l'homme ${ }^{23}$. R. Markus a souligné l'inversion du rapport de dignité entre ces deux formes de vie au $v^{e}$ siècle. Selon nous, il est cependant possible de préciser le sens de la raison qu'il attribue à ce processus. Pour lui, cette inversion provient d'une prise de conscience de la difficulté extrême du mode de vie érémitique ${ }^{24}$. Or, Athanase lui-même avait déjà évoqué cette difficulté ${ }^{25}$, ce qui ne l'avait pas empêché d'enjoindre aux hommes d'adopter ce mode de vie. Nous avons donc fait l'hypothèse que la raison de la promotion du cénobitisme se trouvait moins 
dans la prise de conscience de la difficulté du chemin, que dans celle de la faiblesse de l'homme. Cette promotion est contemporaine de la naissance du défaillantisme. Or, cette façon de penser le sujet humain souligne la faiblesse irréductible de l'homme. Aussi, incapable de se gouverner correctement lui-même, l'homme ne peut accéder au salut en demeurant seul. Le cénobitisme apparaît alors comme une solution adéquate à la question du mode de vie permettant d'accéder à Dieu. L'isolement de la communauté permet de ne pas devenir esclave des sollicitations mondaines ${ }^{26}$, tandis que la présence des autres moines permet de compenser en partie la faiblesse naturelle de l'homme ${ }^{27}$.

Circonscrire le perfectionnisme et le défaillantisme permet donc de mieux comprendre la lutte de prestige entre l'érémitisme et le cénobitisme à la fin de l'Antiquité. Mais là n'est pas le seul intérêt de ce travail. Il permet aussi d'expliquer l'évolution du traitement d'un ensemble d'institutions mondaines par les auteurs chrétiens.

\section{Le sujet face aux institutions : l'exemple du mariage}

7 Dans le sujet, la libido, telle qu'elle a été pensée par Augustin durant la controverse pélagienne, est une poussée, qui, si elle n'est pas canalisée, peut mettre en danger le sujet et la communauté ${ }^{28}$. Par conséquent, elle doit être canalisée par des institutions coercitives. Dans le perfectionnisme, rien de tel : puisque l'homme peut parvenir seul au salut, toute liaison au monde vaut comme compromission. Cela peut expliquer le privilège accordé à l'état de virginité et au célibat comparé à l'état marital. La virginité et le célibat valent chez les auteurs chrétiens qui en soulignent le prestige pour deux choses: le sujet célibataire n'est pas esclave des sollicitations mondaines caractéristiques du mariage, le sujet vierge manifeste en son corps un état de pureté parfait. Un sujet célibataire et vierge, c'est un sujet pleinement disponible à Dieu comme l'exprime clairement Méthode d'olympe (seconde moitié du III $^{\mathrm{e}}$ siècle) dans le Banquet ${ }^{29}$. L'évolution augustinienne concernant le traitement de la question de la dignité respective de la virginité et du mariage est contemporaine de l'évolution de son anthropologie du perfectionnisme au défaillantisme: on le voit tour à tour rejeter le mariage comme impropre au statut du sage ${ }^{30}$ et le défendre dans la mesure où il permet à l'homme de compenser sa faiblesse naturelle ${ }^{31}$. Autrement dit, la présence en l'homme d'une pulsion irréductible légitime ontologiquement sa soumission à des institutions coercitives dont le mariage est une occurrence.

\section{Perspectives}

Ce travail de doctorat est une étape d'un projet plus global : mettre en évidence les modalités de justification de l'exercice des pouvoirs religieux et civils à la fin de l'Antiquité et au début du Moyen Âge. Il s'agit maintenant pour nous d'évaluer la façon dont les discours christologiques et anthropologiques se sont croisés durant cette période. Comme cela nous est en effet apparu clairement, choisir, comme Augustin l'a fait face aux pélagiens, de faire de l'œuvre rédemptrice du Christ une pièce maîtresse de l'enseignement chrétien conduit à revoir les capacités humaines à la baisse. Si, de ce point de vue, le concile de Carthage de 418 a entériné les positions augustiniennes ${ }^{32}$, il reste à mesurer précisément les modalités selon lesquelles, à partir de cette date, christologie et anthropologie se sont trouvées liées. Cette liaison n'est pas anecdotique puisque, si on suit la leçon de Spinoza, toute forme d'exercice du pouvoir se fonde sur 
une anthropologie qui la légitime. De la sorte, il semble que l'étude de l'institutionnalisation du christianisme au Moyen Âge ${ }^{33}$ et des relations entre le pouvoir civil et le pouvoir religieux puisse gagner en précision à partir d'une prise en compte de ce moment de la pensée chrétienne où le défaillantisme a supplanté le perfectionnisme ${ }^{34}$.

Reçu : 30 juillet 2014 - Accepté : 24 novembre 2014

\section{NOTES}

1. Nous entendons par «anthropologie » une forme contingente de représentation de la nature humaine, c'est-à-dire une conception spécifique, d'une part, de la structure de la nature de l'homme renfermant une réflexion relative aux propriétés et aux capacités humaines, et, d'autre part, de la place de l'homme dans l'économie générale du monde. Nous nous inspirons pour cela, en les modifiant en partie, des réflexions de Louis Gernet qui définissait, dans Anthropologie de la Grèce antique, l'anthropologie comme « la représentation de l'être humain sur le plan religieux et de la place qui lui est assignée dans une économie religieuse du monde » (Paris, 1982, p. 13).

2. Sur ce point, voir M. Foucault, Sécurité, territoire, population, Paris, 2004 et P. Chevallier, "Étudier l'Église comme gouvernementalité », Bulletin du centre d'études médiévales d'Auxerre, Hors-série $n^{\circ} 7$ (2013), URL : http://cem.revues.org/12874; DOI : 10.4000/cem.12874.

3. En suivant l'intuition de Spinoza dans le Traité politique (trad. C. APPUHN, Paris, 1966, § 4).

4. M. FOUCAUlT, Sécurité, territoire..., op. cit., p. 168.

5. Dans le chapitre 6 de Les Sources du Moi. La formation de l'identité moderne (trad. C. MELANçoN, Paris, 1998), C. Taylor met clairement en évidence, à partir d'une étude détaillée de l'éthique platonicienne, que l'ensemble des grandes écoles philosophiques antiques partagent, malgré leurs différences apparentes, deux idées fondamentales : d'une part, le cheminement existentiel du sujet est polarisé par l'idéal de maîtrise de soi, d'autre part, la nature de l'homme est telle qu'il est en droit possible pour chaque être de réaliser en cette vie cet idéal.

6. R. MARKUS, " "Vous serez parfaits" ", in Au risque du christianisme. L'émergence du modèle chrétien ( $V^{e}-V^{e}$ siècle), trad. D. KEMPF, Lyon, 2012, p. 105-131.

7. Ainsi, chez Platon comme chez Aristote, le vice et la vertu ne sont pas des données structurelles de la nature humaine, même si leur possibilité existe en nous naturellement. Mais, par nature, le sujet n'est ni vicieux ni vertueux. Il ne le devient que par habitude, de sorte que la réflexion politique implique nécessairement une réflexion portant sur l'éducation. Pour le traitement de cette question chez Platon, voir Les Lois, trad. L. BRISSON et J.-F. PRADEAU, Paris, 2006, VII, 788a-c ; chez Aristote, voir Éthique à Nicomaque, trad. J. TRICOT, Paris, 1997, II, 1.

8. ATHANASE D'AlEXANDRIE, Vie d'Antoine, trad. G. J. M. BARTELINK, Paris, 1994 (Sources chrétiennes, 400), par exemple 5, 1-6.

9. ATHANASE D'AlEXANDRIE, Vie d'Antoine..., ibid., 20, 1-6.

10. Voir notamment l'Histoire lausiaque de Pallade d'Hélénopolis (trad. N. MOLINIER, Bégrolles-enMauge, 1999 [Spiritualité orientale, 75]), l'Histoire des moines d'Égypte (trad. A.-J. FESTUGIÈRE, dans Les Moines d'Orient, Paris, t. 4, 1964) et les Apophtegmes des Pères (trad. J.-C. GUY, 3 vol., Paris, 1993, 2003 et 2005 [Sources chrétiennes, 387, 474 et 498]). 
11. AUgustin, Confessions, trad. E. TRÉHOREL et G. BOUISSOU, Paris, 1992 (Bibliothèque augustinienne, 13), VIII, V, 10 : «De la volonté perverse naît la passion [ex uoluntate perversa facta est libido], de l'esclavage de la passion naît l'habitude [et dum seruitur libidini facta est consuetudo] et de la nonrésistance à l'habitude naît la nécessité [et dum consuetudini non resistitur facta est necessitas]. »

12. Augustin, Cité de Dieu, trad. G. comBÈs, Paris, 1959 (Bibliothèque augustinienne, 35), XIV, XVXIX.

13. Augustin, Cité de Dieu..., ibid., XIV, XVI-XIX. Sur ce point, voir FoucAult, «Sexualité et solitude ", in Dits et écrits (II), texte $n^{\circ}$ 295, Paris, 2001, p. 987-997. Dans cet article, les réflexions foucaldiennes concernant le dispositif liant l'examen de soi et l'aveu dans le contexte monastique chrétien antique se fondent sur ce que nous avons nommé «défaillantisme ». Le fait que le pastorat chrétien fasse peser sur le sujet des injonctions permanentes est légitimé par l'infirmité naturelle de l'homme : puisque, par nature, il ne peut correctement se gouverner lui-même, alors il doit sans cesse se soumettre, en l'occurrence il doit sans cesse s'examiner et avouer. Et ce qu'il doit examiner, ce sont les manifestations de cette altérité enracinée en lui qu'Augustin pense à partir du concept de «libido». Autrement dit, l'« herméneutique de soi », que Foucault repère dans la direction de conscience chrétienne antique, est une herméneutique de la défaillance.

14. Cette controverse dure de 411 à 430, date de la mort d'Augustin. Pélage (vers 350-420) soutenait que le sujet pouvait parvenir au salut grâce à ses propres moyens, adoptant là une perspective perfectionniste déjà ancienne, dont son disciple Julien d'Éclane (vers 386-455) exposa toutes les conséquences face à Augustin. Si le milieu ascétique chrétien ne trouvait rien à redire à cette idée, Augustin en saisit rapidement le danger. En effet, si l'accès au salut peut être l'œuvre propre du sujet, alors l'œuvre rédemptrice du Christ s'avère inutile. Si l'homme a besoin de la grâce divine pour être sauvé, et pour Augustin cela est une donnée scripturaire impossible à remettre en cause, c'est qu'il est, par nature, infirme. La conception augustinienne de la libido comme pôle d'altérité logé au cœur même du sujet a ainsi pour fonction de clairement rendre compte de cette infirmité. M. Lamberigts offre un exposé clair de l'opposition entre Augustin et Julien d'Éclane concernant la question de la structure de la nature humaine dans «Julien d'Éclane et Augustin : deux conceptions d'Adam ", in Augustiniana, 40 (1990), p. 373-410.

15. Voir notamment JEAN CASSIEN, Institutions cénobitiques, trad. J.-C. GUY, Paris, 2011 (Sources chrétiennes, 109), IV, 39, 2.

16. JEAN CASSIEN, Institutions cénobitiques..., ibid., IV, 39, 2.

17. Voir, par exemple, le Prologue de la Règle de saint Benoît.

18. Voir le chapitre 73 de la règle bénédictine conseillant vivement la lecture de Cassien.

19. Cassien associe ainsi, dans les Institutions cénobitiques, la « règle commune » à « l'exemple des anciens » [maiorum exempla] $(\mathrm{IV}, 39,2)$.

20. Sur ce point, voir R. MARKUS, « Désert et cité : des frontières qui s'estompent », in Au risque du christianisme..., op. cit., p. 247-267.

21. Cette proportionnalité inverse entre la liaison au monde et la disponibilité à Dieu se trouve déjà, pensée à partir d'autres concepts, chez Plotin. Voir notamment Traité 51 (I, 8), 4, 25-31, trad. L. LAVAUD, in PLOTIN, Traités 51-54, Paris, 2010.

22. ATHANASE D'ALEXANDRIE, Vie d'Antoine, 2, 3. La citation est extraite de Mt 19, 21.

23. Concernant la question du genre de vie le plus adéquat pour faire la volonté de Dieu, la tension entre érémitisme et cénobitisme est clairement perceptible dans la Préface des Conférences de Cassien (trad. E. PICHERY, Paris, 2008 [Sources chrétiennes, 42bis]).

24. R. MARKUS, Au risque du christianisme..., op. cit., p. 249-252.

25. ATHANASE D'ALEXANDRIE, Vie d'Antoine, 20, 1-2.

26. Voir, par exemple, JEAN CASSIEN, Institutions cénobitiques, IV, 13-14 ; 34-36.

27. Voir, par exemple, les chapitres 71 et 72 de la Règle de saint Benoît. Que l'infirmité humaine soit irréductible ne signifie pas qu'elle soit irrépressible. Son irréductibilité lui vient, dans le 
contexte chrétien, du fait que seul Dieu a le pouvoir de transformer la nature. Or, l'infirmité de l'homme est la conséquence d'une restructuration de la nature humaine après le péché originel. De la sorte, l'accès à l'autonomie requiert l'action gracieuse de Dieu. Malgré cela, il est possible, au moyen d'un ensemble d'institutions spécifiques, de compenser l'infirmité humaine en canalisant l'action de la pulsion. La direction de conscience, dans sa structuration spécifiquement chrétienne, est l'une de ces institutions. Sur ce point, voir M. Foucault, Du gouvernement des vivants, Paris, 2012, leçons du 12, du 19 et du 26 mars 1980.

28. Preuve de la pérennité de cette façon de penser la nature humaine, S. Freud, en faisant du «caractère poussant» le propre de la pulsion («Pulsions et destin des pulsions», in Métapsychologie, trad. J. Laplanche et J.-B. Pontalis, Paris, 1968, p. 17-18), soulignera ses dangers à la fois au niveau ontogénétique («Le refoulement», in Métapsychologie, op. cit.) et au niveau phylogénétique (Malaise dans la civilisation, trad. A. Weill, Paris, 2010). La reconstitution du défaillantisme chrétien antique n'est donc pas à elle-même sa seule fin : elle permet d'historiciser la façon dont, nous-mêmes, nous pensons la nature humaine.

29. MÉTHODE D’olyMPE, Le Banquet, trad. V.-H. DEBIDOUR, Paris, 1963 (Sources chrétiennes, 95), 10, I, 258.

30. AUgustin, Soliloques, dans Problèmes moraux (II), trad. P. DE LABRIOLLE, Paris, 1948 (Bibliothèque augustinienne, 5), I, 17.

31. AUGUSTIN, Le Bien du mariage, in Problèmes moraux, trad. G. ComBÈs, Paris, 1948 (Bibliothèque augustinienne 2), VI, 6 .

32. Voir l'introduction de $\mathrm{C}$. Munier au volume 22 de la Bibliothèque augustinienne (La Crise pélagienne II, Paris, 1975, p. 9-24).

33. Il y a ainsi fort à parier que l'étude de la pratique de la direction de conscience et de la confession gagne en précision en tenant compte des spécificités du défaillantisme né à la fin de l'Antiquité. Ici, comme souvent, on perçoit que l'historien et l'historien des idées ont grand intérêt à travailler ensemble.

34. Bien qu'il faille noter que l'émergence du défaillantisme au début du ve siècle n'implique pas une disparation du perfectionnisme. Il semble en effet que nombre des mouvements, qui, dans le christianisme postérieur, ont inspiré à Foucault la notion de "contre conduite" (Sécurité, territoire..., op. cit., leçon du $1^{\mathrm{er}}$ mars 1978), peuvent être lus dans le cadre de l'opposition de ces deux modalités de conception du sujet humain.

\section{INDEX}

Mots-clés : perfection, défaillance, salut, christianisme antique

\section{AUTEUR \\ ANTHONY MANICKI}

Laboratoire Triangle - UMR 5206 